\title{
Non-Commutative Geometry and Measurements of Polarized Two Photon Coincidence Counts
}

\author{
S. Sivasubramanian, G. Castellani, N. Fabiano ${ }^{\dagger}$, \\ A. Widom, J. Swain, Y.N. Srivastava ${ }^{\dagger}$, G. Vitiello ${ }^{\dagger \dagger}$ \\ Physics Department Northeastern University, Boston MA USA \\ ${ }^{\dagger}$ Physics Department \& INFN, University of Perugia, Perugia Italy \\ $\dagger$ Physics Department \& INFN, University of Salerno, Salerno Italy
}

\begin{abstract}
Employing Maxwell's equations as the field theory of the photon, quantum mechanical operators for spin, chirality, helicity, velocity, momentum, energy and position are derived. The photon "Zitterbewegung" along helical paths is explored. The resulting non-commutative geometry of photon position and the quantum version of the Pythagorean theorem is discussed. The distance between two photons in a polarized beam of given helicity is shown to have a discrete spectrum. Such a spectrum should become manifest in measurements of two photon coincidence counts. The proposed experiment is briefly described.
\end{abstract}

\section{Introduction}

In recent years there has been considerable mathematical and physical interest in noncommutative geometry[1] and its physical consequences. Perhaps, the most simple example of a non-commutative geometry consists of the geometric plane described by Hermitian operator coordinates $(\hat{X}, \hat{Y})$ that do not commute; e.g. there exists an area $\mathcal{A}$ such that

$$
i[\hat{X}, \hat{Y}]=\mathcal{A} \text {. }
$$

As a consequence of Eq.(1), there is a "quantization" of the Pythagorean theorem in that the distance $\hat{D}=\sqrt{\hat{X}^{2}+\hat{Y}^{2}}$ has a quantized spectrum[2]. For $n=0,1,2,3, \ldots$, we have

$$
\left(\hat{X}^{2}+\hat{Y}^{2}\right)|n\rangle=D_{n}^{2}|n\rangle \text { where } D_{n}=\sqrt{(2 n+1) \mathcal{A}} .
$$

Experimental examples of physical systems which can be usefully described by coordinates in the non-commutative plane include the following: (i) vortices in superfluid ${ }^{4} \mathrm{He}$ films[3], (ii) quantum Hall effect charged magnetic vortices in two-dimensional electron liquids [4], (iii) quantum interference phase between two alternative paths in the plane (as in the Aharonov-Bohm effect)[2] and (iv) high energy charged lepton 
beams stored in cyclotrons [5, 6, 7]. In all of these experimental examples, there exists the ordinary Euclidean geometry of position vectors $\left(\mathbf{r}_{1}, \mathbf{r}_{2}, \ldots\right)$ of the particles which make up the system. The Euclidean coordinates may appear in the quantum mechanical wave functions of the system. Euclidean coordinates may also appear in the classical rulers employed to construct the lengths scales of the apparatus used in measuring the quantum mechanical system properties. However, the quantum coordinates (such as vortex positions or the positions of cyclotron orbit centers) are described by strictly non-commuting operators. Thus, both classical Euclidean geometry and quantum non-commutative geometry live tranquilly together in the theoretical description of the above experimental quantum systems.

Such tranquility also exists in the quantum mechanical behavior of zero mass nonzero spin quantum mechanical particle beams. These include neutrinos, photons $[8]$ and gravitons. Our purpose is to discuss the case of zero mass photons [9, 10, 11, 12, 13, [14], where the experimental technology[15] (of quantum optics) is most fully developed. Our central theoretical results concern two photon coincidence counts measured from polarized beams. The statistics of the counts should reflect the non-commutative geometry of the photon positions.

In Sec 2 we write Maxwell's equations in the vacuum in a form conducive to describing the photon as a spin one object. The quantum mechanical spin, chirality, helicity, velocity, momentum and energy operators for a single photon will be derived. In Sec 3 the "velocity" operator of the photon will be explored. Writing the photon velocity operator as $\dot{\mathbf{r}}=\mathbf{v}=\left(v_{x}, v_{y}, v_{z}\right)$, one finds that each component formally has the possible eigenvalues $-c, 0$ or $+c$. However, each component also has non-trivial commutation relations with the other components finally yielding

$$
\mathbf{v} \cdot \mathbf{v}=v_{x}^{2}+v_{y}^{2}+v_{z}^{2}=2 c^{2}>c^{2} \text { (photons). }
$$

Eq.(3) indicates a considerable amount of "Jitterbugging" in the photon motion. For a Dirac particle, wherein $\mathbf{v}_{\text {Dirac }} \cdot \mathbf{v}_{\text {Dirac }}=3 c^{2}$, Schrödinger called the resulting (superluminal) velocity fluctuations Zitterbewegung (German Jitterbugging). In more detail, it will be shown that the component of photon velocity perpendicular to the photon momentum $\mathbf{p}$ undergoes a rotation at angular velocity $\Omega=c|\mathbf{p}| / \hbar$. The resulting photon "path" may then be described as helical. Along the axis of the helix, the photon moves with velocity $c$. But the photon also moves around the helix in such a way that the total speed is greater than $c$. In Sec 4 the Wigner photon position operator will be discussed. The photon position will be shown to obey the rules of a non-commutative geometry with an associated quantum Pythagorean theorem. The motivation for introducing such a position operator can be understood in terms of the photon angular momentum as is discussed in Sec 5 In Sec 6 the non-commutative geometry of a two photon system will be discussed. The distance between two photons in a polarized beam is shown to have a discrete spectrum. Such a spectrum should become manifest in measurements of two photon coincidence counts. In the concluding Sec 7 the technology of these proposed experiments will be briefly described. 


\section{Maxwell's Equations}

The Maxwell equations describing a photon in the vacuum are well known. The relativistic covariance is well established so that we pick a particular inertial frame (say the "laboratory frame") in which they read

$$
\begin{aligned}
\operatorname{div} \mathbf{E} & =0, \\
\operatorname{div} \mathbf{B} & =0, \\
\operatorname{cur} l \mathbf{E} & =-\frac{1}{c}\left(\frac{\partial \mathbf{B}}{\partial t}\right), \\
\operatorname{cur} l \mathbf{B} & =\frac{1}{c}\left(\frac{\partial \mathbf{E}}{\partial t}\right) .
\end{aligned}
$$

Introducing the complex electromagnetic fields $\mathbf{F}_{+}$and $\mathbf{F}_{-}$via

$$
\mathbf{F}_{ \pm}=\mathbf{E} \pm i \mathbf{B}
$$

allows us to write Maxwell's equations in the more compact form

$$
\operatorname{div} \mathbf{F}_{ \pm}=0 \text { and } i\left(\frac{\partial \mathbf{F}_{ \pm}}{\partial t}\right)= \pm c \operatorname{curl} \mathbf{F}_{ \pm} .
$$

The operator curl defines the spin one matrices $\mathbf{S}=\left(S_{x}, S_{y}, S_{z}\right)$ according to the equivalent definitions

$$
\mathbf{U}=\operatorname{curl} \mathbf{W}
$$

and

$$
\left(\begin{array}{c}
U_{x} \\
U_{y} \\
U_{z}
\end{array}\right)=\left(\begin{array}{ccc}
0 & -\partial_{z} & \partial_{y} \\
\partial_{z} & 0 & -\partial_{x} \\
-\partial_{y} & \partial_{x} & 0
\end{array}\right)\left(\begin{array}{l}
W_{x} \\
W_{y} \\
W_{z}
\end{array}\right)=-i(\mathbf{S} \cdot \mathbf{g r a d})\left(\begin{array}{c}
W_{x} \\
W_{y} \\
W_{z}
\end{array}\right) .
$$

Eq.(8) implies the explicit representation

$$
S_{x}=\left(\begin{array}{ccc}
0 & 0 & 0 \\
0 & 0 & -i \\
0 & i & 0
\end{array}\right), \quad S_{y}=\left(\begin{array}{ccc}
0 & 0 & i \\
0 & 0 & 0 \\
-i & 0 & 0
\end{array}\right), \quad S_{z}=\left(\begin{array}{ccc}
0 & -i & 0 \\
i & 0 & 0 \\
0 & 0 & 0
\end{array}\right) .
$$

Note that the usual spin commutation relations,

$$
\left[S_{x}, S_{y}\right]=i S_{z}, \quad\left[S_{y}, S_{z}\right]=i S_{x}, \quad\left[S_{z}, S_{x}\right]=i S_{y},
$$

hold true as well as

$$
S_{x}^{2}+S_{y}^{2}+S_{z}^{2}=S(S+1) \text { where } S=1 .
$$

Introducing the complex column three vectors $F_{+}$and $F_{-}$via

$$
F_{ \pm}=\left(\begin{array}{c}
E_{x} \pm i B_{x} \\
E_{y} \pm i B_{y} \\
E_{z} \pm i B_{z}
\end{array}\right)
$$


yields Maxwell's equations in the form

$$
i \frac{\partial F_{ \pm}}{\partial t}=\mp i c(\mathbf{S} \cdot \mathbf{g r a d}) F_{ \pm}
$$

where Eqs. (6), (7) and (8) have been invoked. Eq. (13) describes (in both righthanded $F_{+}$and lefthanded $F_{-}$representations) the vacuum field equations for spin $S=1$ massless photons.

To see more clearly what is involved, let us consider the case of massless spin one-half particles, i.e. the Weyl massless neutrinos. Defining the momentum operator as

$$
\mathbf{p}=-i \hbar \text { grad }
$$

and with $\sigma=\left(\sigma_{x}, \sigma_{y}, \sigma_{z}\right)$ denoting the Pauli matrices,

$$
\sigma_{x}=\left(\begin{array}{cc}
0 & 1 \\
1 & 0
\end{array}\right), \quad \sigma_{y}=\left(\begin{array}{cc}
0 & -i \\
i & 0
\end{array}\right), \quad \sigma_{z}=\left(\begin{array}{cc}
1 & 0 \\
0 & -1
\end{array}\right),
$$

the massless "Weyl neutrino" equations read

$$
\begin{array}{lll}
i \hbar \frac{\partial \psi_{+}}{\partial t} & =c(\sigma \cdot \mathbf{p}) \psi_{+} & \text {righthanded Weyl } \\
i \hbar \frac{\partial \psi_{-}}{\partial t} & =-c(\sigma \cdot \mathbf{p}) \psi_{-} & \text {lefthanded Weyl. }
\end{array}
$$

The photon analogues to Eqs.(16) are

$$
\begin{array}{ll}
i \hbar \frac{\partial F_{+}}{\partial t}=c(\mathbf{S} \cdot \mathbf{p}) F_{+} & \text {righthanded Maxwell, } \\
i \hbar \frac{\partial F_{-}}{\partial t}=-c(\mathbf{S} \cdot \mathbf{p}) F_{-} & \text {lefthanded Maxwell. }
\end{array}
$$

There is a difference between the spin one half Eqs. (16) and the spin one Eqs.117. A lefthanded and a righthanded neutrino are different particles. Only lefthanded neutrinos have so far appeared in the laboratory which is a basis for parity violation. In spite of the wide spread theoretical righthanded cross product convention for writing down Maxwell's equations, electromagnetic theory is inherently parity symmetry invariant. Nevertheless, it is useful to construct a six component field column vector[16] out of the three component righthanded and lefthanded column vectors defined in Eq.[12); i.e.

$$
F=\left(\begin{array}{l}
F_{+} \\
F_{-}
\end{array}\right)
$$

The operators for the photon are now in part described by $6 \times 6$ matrices naturally constructed by partitioned $3 \times 3$ sub-matrices. For example, employing the $3 \times 3$ spin matrices in Eq. (9) we may write for the $6 \times 6$ spin one matrices

$$
\boldsymbol{\Sigma}=\left(\begin{array}{ll}
\mathbf{S} & 0 \\
0 & \mathbf{S}
\end{array}\right)
$$

The photon chirality matrix may be defined as

$$
\Gamma_{5}=\left(\begin{array}{cc}
1 & 0 \\
0 & -1
\end{array}\right) .
$$


The chiral $\Gamma_{5} \operatorname{spin} \Sigma$ matrix product

$$
\alpha=\Gamma_{5} \boldsymbol{\Sigma}=\left(\begin{array}{cc}
\mathbf{S} & 0 \\
0 & -\mathbf{S}
\end{array}\right),
$$

is useful for describing the massless photon Hamiltonian

$$
\mathcal{H}=c(\alpha \cdot \mathbf{p}) .
$$

Eqs. (17), (18), 21) and and (22) imply the photon (Maxwell) wave equation in the Schrödinger-Dirac form

$$
i \hbar \frac{\partial F}{\partial t}=\mathcal{H} F .
$$

To obtain the energy eigenvalues for the photon, one needs to solve

$$
\mathcal{H} F_{\mathbf{p}}(\mathbf{r})=\tilde{\epsilon}(\mathbf{p}) F_{\mathbf{p}}(\mathbf{r}) .
$$

Choosing $F_{\mathbf{p}}(\mathbf{r})=F_{\mathbf{p}}(0) e^{i \mathbf{p} \cdot \mathbf{r} / \hbar}$ yields the energy eigenvalues

$$
\begin{array}{lll}
\tilde{\boldsymbol{\epsilon}}_{+}(\mathbf{p})=c|\mathbf{p}| & \text { (photon), } \\
\tilde{\boldsymbol{\epsilon}}_{-}(\mathbf{p})=-c|\mathbf{p}| & \text { (anti }- \text { photon), } \\
\tilde{\boldsymbol{\epsilon}}_{0}(\mathbf{p})=0 & \text { (forbidden), }
\end{array}
$$

where the anti-photon (negative energy state) is merely the photon moving backward in time. For $\mathbf{p} \neq 0$, the zero eigenvalue of energy is forbidden in virtue of the vacuum Gauss' law $\operatorname{div} \mathbf{E}=0$ and $\operatorname{div} \mathbf{B}=0$. The photon velocity operator follows from the commutator

$$
\mathbf{v}=\frac{i}{\hbar}[\mathcal{H}, \mathbf{r}]=\frac{\partial \mathcal{H}}{\partial \mathbf{p}}=c \alpha=c\left(\Gamma_{5} \mathbf{\Sigma}\right) .
$$

The photon helicity operator $\Lambda$ is conventionally defined as

$$
\Lambda=\left(\frac{\mathbf{p} \cdot \mathbf{\Sigma}}{|\mathbf{p}|}\right),
$$

The allowed eigenvalues of helicity are $\Lambda= \pm 1$ while the eigenvalue $\Lambda=0$ is forbidden by the Gauss law constraints $\operatorname{div} \mathbf{F}_{ \pm}=0$ of Eq. (6). The photon Hamiltonian is given by

$$
\mathcal{H}=(\mathbf{v} \cdot \mathbf{p})=c(\alpha \cdot \mathbf{p})=c \Gamma_{5}(\boldsymbol{\Sigma} \cdot \mathbf{p}),
$$

which can then be expressed directly in terms of chirality $\Gamma_{5}$ and helicity $\Lambda$ as

$$
\mathcal{H}=c|\mathbf{p}| \Gamma_{5} \Lambda .
$$

The eigenvalue spectrum of $\mathcal{H}$ in Eq. 25) follows directly from the representation in Eq. [29]. 


\section{Photon Velocity}

Let us now contemplate how fast a photon is moving. For a photon of momentum $\mathbf{p}$ and energy $\varepsilon(\mathbf{p})=c|\mathbf{p}|$ the mean photon (three vector) velocity is the expected light velocity value

$$
\overline{\mathbf{v}}=\frac{\overline{\partial \mathcal{H}}}{\partial \mathbf{p}}=\frac{\partial \varepsilon}{\partial \mathbf{p}}=c\left(\frac{\mathbf{p}}{|\mathbf{p}|}\right) .
$$

On the other hand, from Eq. 26 we find that photon velocity operator obeys

$$
\mathbf{v} \cdot \mathbf{v}=c^{2}\left(\Gamma_{5} \boldsymbol{\Sigma}\right) \cdot\left(\Gamma_{5} \boldsymbol{\Sigma}\right)=c^{2}\left(\Sigma_{x}^{2}+\Sigma_{y}^{2}+\Sigma_{z}^{2}\right)=c^{2} S(S+1)
$$

where $\left[\Gamma_{5}, \boldsymbol{\Sigma}\right]=0$ and $\left(\Gamma_{5}\right)^{2}=1$ has been invoked. Since the photon has spin $S=1$, it follows that

$$
\mathbf{v} \cdot \mathbf{v}=2 c^{2} .
$$

Thus, the root mean square velocity of a photon is the superluminal value $c \sqrt{2}$. What is the nature of the velocity fluctuations? The answer resides in the operator for the photon acceleration $\mathbf{a}=\dot{\mathbf{v}}$; i.e.

$$
\dot{\mathbf{v}}=\frac{i}{\hbar}[\mathcal{H}, \mathbf{v}]=\frac{i}{\hbar}[(\mathbf{v} \cdot \mathbf{p}), \mathbf{v}] .
$$

Employing

$$
\left[v_{i}, v_{j}\right]=c^{2}\left[\Gamma_{5} \Sigma_{i}, \Gamma_{5} \Sigma_{j}\right]=c^{2}\left[\Sigma_{i}, \Sigma_{j}\right]=i c^{2} \epsilon_{i j k} \Sigma_{k}=i c \Gamma_{5} \epsilon_{i j k} v_{k}
$$

together with Eq. 33. yields the equation of motion for the photon velocity

$$
\dot{\mathbf{v}}=\mathbf{\Omega} \times \mathbf{v} \quad \text { wherein } \quad \hbar \mathbf{\Omega}=c \Gamma_{5} \mathbf{p} .
$$

The physical meaning of Eq. (35) is unambigous. The velocity of the photon precesses about the momentum $\mathbf{p}=\hbar \mathbf{k}$ direction at an angular velocity

$$
\mathbf{\Omega}_{ \pm}= \pm\left(\frac{c \mathbf{p}}{\hbar}\right)= \pm c \mathbf{k}
$$

with the sign depending on the chirality $\Gamma_{5}$ of the photon.

From the classical electromagnetic theory viewpoint, one envisions either a right or left circularly polarized electromagnetic wave with frequency $\Omega=c|\mathbf{k}|$. From a photon viewpoint, with $\mathbf{p}=\hbar \mathbf{k}$, the photon moves with velocity $c$ in the direction of $\mathbf{k}$. The photon velocity component perpendicular to the $\mathbf{k}$-direction is rotating (in either a right or left handed direction) at an angular velocity $\boldsymbol{\Omega}_{ \pm}= \pm c \mathbf{k}$. The physical picture is depicted in Fig 1

In position space, the photon moves on a "helical" path. The velocity component along the axis of the helix is light speed. On the other hand, the photon is going around and around on a helix with a total superluminal speed of $c \sqrt{2}$. Let us now consider in more detail how the helical path enters into the position representation for the photon. 


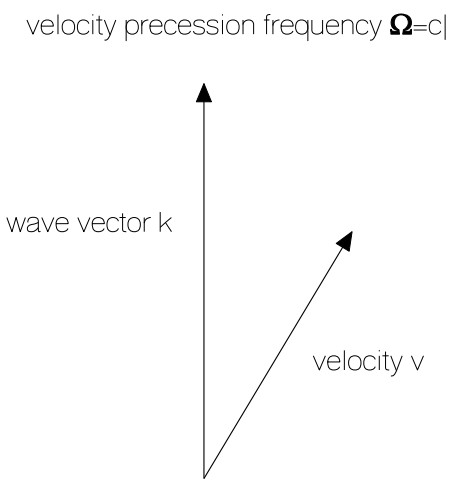

Figure 1: The circularly polarized photon velocity $\mathbf{v}$ precesses about the wave vector $\mathbf{k}=\mathbf{p} / \hbar$ axis at an angular velocity $\boldsymbol{\Omega}_{ \pm}= \pm c \mathbf{k}$ with the sign depending on chirality. The component of the photon velocity parallel to the wave vector $\mathbf{k}$ is light speed; i.e. $c=(\mathbf{v} \cdot \mathbf{k}) /|\mathbf{k}|$.

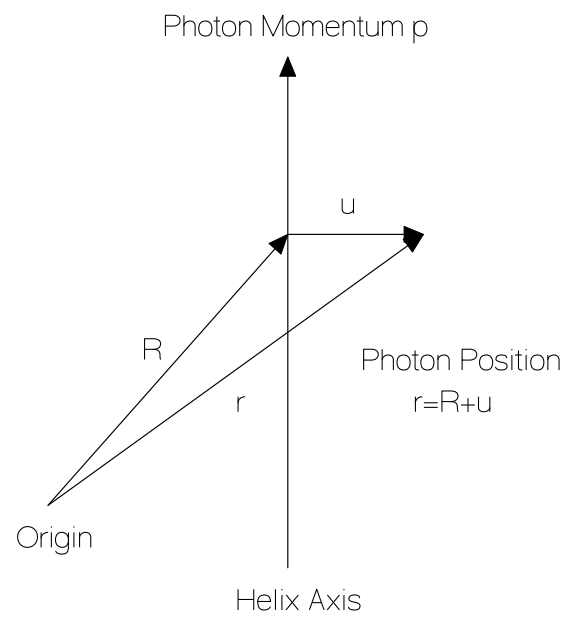

Figure 2: For a photon of momentum $\mathbf{p}=\hbar \mathbf{k}$, we may point the momentum along the axis of a helix. The photon position $\mathbf{r}$ may than be written as a vector $\mathbf{R}$ pointing to the helix axis plus a vector $\mathbf{u}$ normal to the helix axis. The vector $\mathbf{u}$ moves in a circle at angular velocity $\mathbf{\Omega}$ which depends upon the chirality via $\hbar \mathbf{\Omega}=\Gamma_{5} c \mathbf{p}$. 


\section{Photon Position and Helicity}

Consider the position of the photon as an operator in momentum space

$$
\mathbf{r}=i \hbar \frac{\partial}{\partial \mathbf{p}}
$$

The above Eq. 377 implies the usual Heisenberg commutation relations

$$
\left[p_{k}, r_{j}\right]=-i \hbar \delta_{j k} .
$$

The position $\mathbf{r}$ of the photon can be decomposed into a sum of the position $\mathbf{R}$ of the axis of the helix and the displacement $\mathbf{u}$ from the helix axis to the helix coil. As depicted in Fig 2

$$
\mathbf{r}=\mathbf{R}+\mathbf{u},
$$

where

$$
\mathbf{u}=\frac{\hbar(\boldsymbol{\Sigma} \times \mathbf{p})}{|\mathbf{p}|^{2}}=\left(\frac{\hbar \Gamma_{5}}{c}\right) \frac{\mathbf{v} \times \mathbf{p}}{|\mathbf{p}|^{2}} .
$$

Radius vector $\mathbf{u}$ rotates in a circle remaining normal to the photon momentum $\mathbf{p}=\hbar \mathbf{k}$ according to the precession equation of motion

$$
\dot{\mathbf{u}}=\frac{i}{\hbar}[\mathcal{H}, \mathbf{u}]=\mathbf{\Omega} \times \mathbf{u} \quad \text { wherein } \hbar \boldsymbol{\Omega}=c \Gamma_{5} \mathbf{p} .
$$

The radius squared of the helix is then given by

$$
\mathbf{u} \cdot \mathbf{u}=\left(\frac{\hbar}{|\mathbf{p}|}\right)^{2}\left\{\boldsymbol{\Sigma} \cdot \boldsymbol{\Sigma}-\left(\frac{\mathbf{p} \cdot \boldsymbol{\Sigma}}{|\mathbf{p}|}\right)^{2}\right\}=\left(\frac{\hbar}{|\mathbf{p}|}\right)^{2}\left\{S(S+1)-\Lambda^{2}\right\}
$$

For a spin $S=1$ photon with helicity $\Lambda= \pm 1$, Eq.42) implies the square radius of the helix

$$
\mathbf{u} \cdot \mathbf{u}=\left(\frac{\hbar}{p}\right)^{2}=\lambda^{2} \quad \text { (or equivalently) } \sqrt{\mathbf{u} \cdot \mathbf{u}}=|\mathbf{u}|=\lambda
$$

The radius of the helix orbit is completely determined by the wave length $\lambda=(2 \pi \lambda)$. However, the components of the radius vector from the helix axis to the helix path do not commute among themselves. In detail, Eq.40) implies

$$
\left[u_{j}, u_{k}\right]=i\left(\frac{\hbar}{|\mathbf{p}|}\right)^{2} \Lambda \epsilon_{j k l}\left(\frac{p_{l}}{|\mathbf{p}|}\right) .
$$

For example, if a photon moves with a fixed momentum $\mathbf{p}=(0,0, \hbar / \lambda)$ along the $z$-axis with helicity $\Lambda=1$, then the components of $\mathbf{u}$ do not commute $\left[u_{x}, u_{y}\right]=i \lambda^{2}$. Quantum mechanically, the radius of the helix is fixed at $\left(u_{x}^{2}+u_{y}^{2}\right)=\lambda^{2}$ but one cannot tell the values separately of the non-commuting components $\mathbf{u}=\left(u_{x}, u_{y}, 0\right)$.

From Eqs.(34), (38), (40) and (44) it follows that $\left[R_{i}, u_{j}\right]=\left[r_{i}-u_{i}, u_{j}\right]=0$, which together with $\left[r_{i}, r_{j}\right]=0$ implies the non-commutative geometry of the coordinates $\mathbf{R}$; i.e.

$$
i\left[R_{j}, R_{k}\right]=\left(\frac{\hbar}{|\mathbf{p}|}\right)^{2} \Lambda \epsilon_{j k l}\left(\frac{p_{l}}{|\mathbf{p}|}\right) .
$$


If a photon moves with a fixed momentum $\mathbf{p}=(0,0, \hbar / \lambda)$ along the $z$-axis with helicity $\Lambda=1$, then the components of $\mathbf{R}=(X, Y, Z)$ do not commute $[X, Y]=-i \lambda^{2}$ and the quantum Pythagorean theorem of Eq.(2) reads $\sqrt{X^{2}+Y^{2}}|n\rangle=\lambda \sqrt{2 n+1}|n\rangle$ where $n=0,1,2,3, \ldots$.

\section{Photon Angular Momentum}

The non-commutative geometry of the Wigner photon coordinate $\mathbf{R}$ can be motivated purely by considerations relating to the photon angular momentum $\mathbf{J}$. As is usual one writes the total angular momentum as a sum of the orbital angular momentum and the spin angular momentum

$$
\mathbf{J}=\mathbf{r} \times \mathbf{p}+\hbar \mathbf{\Sigma} .
$$

For a massless particle such as the photon, one seeks to express express the total angular momentum in terms of helicity. One employs the identity

$$
\mathbf{p} \times(\boldsymbol{\Sigma} \times \mathbf{p})=|\mathbf{p}|^{2}\left\{\boldsymbol{\Sigma}-\left(\frac{\mathbf{p}}{|\mathbf{p}|}\right) \Lambda\right\}
$$

where the helicity $\Lambda$ is defined in Eq.(27). Eqs.(46) and (47) imply

$$
\mathbf{J}=\mathbf{r} \times \mathbf{p}-\mathbf{u} \times \mathbf{p}+\hbar\left(\frac{\mathbf{p}}{|\mathbf{p}|}\right) \Lambda .
$$

where $\mathbf{u}$ is defined in Eq. (40). If we employ the Wigner photon position

$$
\mathbf{R}=\mathbf{r}-\mathbf{u}=\mathbf{r}-\left\{\frac{\hbar(\boldsymbol{\Sigma} \times \mathbf{p})}{|\mathbf{p}|^{2}}\right\}
$$

then the total angular momentum may be written in terms of the proper (zero mass particle) orbital angular momentum $\mathbf{L}=\mathbf{R} \times \mathbf{p}$ and the helicity $\Lambda$ according to

$$
\begin{array}{rlr}
\mathbf{J} & =\mathbf{L}+\hbar \boldsymbol{\Sigma}_{\Lambda} \equiv \mathbf{R} \times \mathbf{p}+\hbar\left(\frac{\mathbf{p}}{|\mathbf{p}|}\right) \Lambda, \\
\Lambda & = \pm 1 & \text { (allowed helicity), } \\
\Lambda & =0 & \text { (forbidden helicity) } .
\end{array}
$$

It is the Wigner photon coordinate which gives rise to a non-commutative geometry via

$$
\begin{aligned}
{\left[R_{j}, R_{k}\right] } & =-i \mathcal{A}_{j k} \\
\mathcal{A}_{j k} & =\left(\frac{\hbar}{|\mathbf{p}|}\right)^{2} \Lambda \epsilon_{j k l}\left(\frac{p_{l}}{|\mathbf{p}|}\right) \equiv \epsilon_{j k l} A_{l} .
\end{aligned}
$$

The axial vector area,

$$
\mathbf{A}=\hbar^{2}\left(\frac{\mathbf{p} \Lambda}{|\mathbf{p}|}\right) \text { wherein }|\mathbf{p}|=\frac{\hbar}{\hbar}
$$

plays an important role in the technology of photon detectors as will be discussed in what follows. The main physical point is that the position of a photon in the plain normal to the momentum is only defined within an area $|\mathbf{A}|=\hbar^{2}=|\mathbf{u}|^{2}$. 


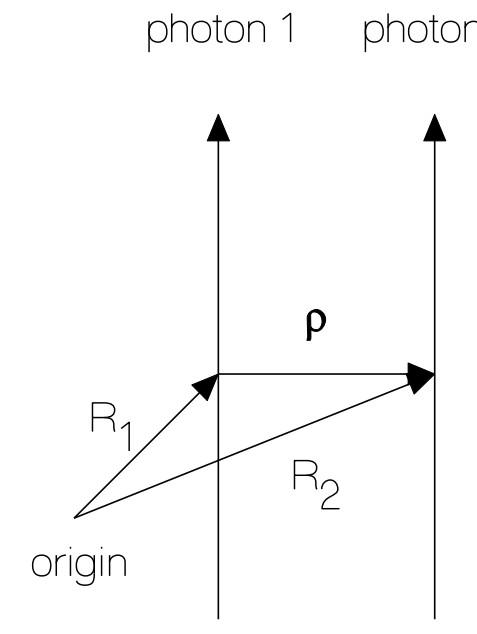

Figure 3: Two photons with the same momentum and helicity, move along different helical paths described by different Wigner coordinate vectors $\mathbf{R}_{1}$ and $\mathbf{R}_{2}$. The coordinate vector $\rho=\mathbf{R}_{2}-\mathbf{R}_{1}$ describes the displacement vector from one helical axis to the other helical axis.

\section{Two Photon States}

Consider two photons each with the same momentum $\mathbf{p}$ and helicity $\Lambda$. Let $\mathbf{R}_{1}$ and $\mathbf{R}_{2}$ represent, respectively, the Wigner positions of the first and second photon. Finally, let

$$
\rho=\mathbf{R}_{2}-\mathbf{R}_{1}
$$

represent the vector displacement from the helical axis of the first photon to the helical axis of the second photon as shown in Fig 3 From Eqs. 51, (52) and 53) it follows that the displacement between helical axes obeys the non-commutative geometry rule

$$
\left[\rho_{j}, \rho_{k}\right]=-i \epsilon_{j k l}\left(A_{12}\right)_{l} \quad \text { with area } \quad \mathbf{A}_{12}=\left(\frac{\hbar^{2} \mathbf{p}_{1} \Lambda_{1}}{p_{1}^{3}}\right)+\left(\frac{\hbar^{2} \mathbf{p}_{2} \Lambda_{2}}{p_{2}^{3}}\right) .
$$

The distance between the two photon helical axes is then quantized in units of $\lambda$ according to

$$
\begin{aligned}
\mathbf{p}_{1}=\mathbf{p}_{2} & =(0,0, \hbar / \hbar), \\
\left(\rho_{x}^{2}+\rho_{y}^{2}\right)|n\rangle & =2 \hbar^{2}(2 n+1)|n\rangle, \\
n & =0,1,2,3, \ldots .
\end{aligned}
$$

Now let us consider a monochromatic beam of photons of a given helicity incident normal to a plane of photon detectors. Let us also consider two photon co- 
incidence counts for the photon detectors in the the plane. According to our central non-commutative geometry Eq. (55), the Wigner distance between the two photon coincidence counts should be quantized into spectrum $D_{n}=\lambda \sqrt{2(2 n+1)}$ where $n=0,1,2,3, \ldots$. Coincidence counting peaks at detector separations $\left\{D_{n}\right\}$ should be observable in the laboratory if the "pixel size" of the photon detectors are small on the scale of $\lambda=(\lambda / 2 \pi)$ where $\lambda$ is the wavelength of the photons.

\section{Conclusion}

Rarely in experiments on optics does one try to resolve the photon position to within a spatial resolution smaller than $\lambda$. For example, in the best commercial digital camera's, the pixel length scale $L>\lambda$ since experimentally it appears difficult to resolve photon positions on a smaller scale. To the authors' knowledge this lower bound on pixel resolution has not been attributed to the non-commutative geometry of photon positions but it does appear likely that such a geometry sets bounds on how well a photon can be localized.

If the photon detectors are of the electric dipole type $L<<\lambda$, and if two photons in a circularly polarized beam are simultaneously measured, then the coincidence count distance $D$ can in principle be measured to a resolution higher than $\lambda$. Observation of peaks spaced at quantized distances $D_{n}=\lambda \sqrt{2(2 n+1)}$ would serve the useful purpose of corroborating the helix picture we have here theoretically obtained.

The question then is: how easy would it be to obtain experimental evidence for the predicted effect? Common position-sensitive devices like charge conductive devices (CCD's) are not sensitive to single photons. Devices suitable for single photon detection based on photocathodes such as photomultipliers or hybrid photodiodes are unlikely to be fabricated with pixels small enough to lie inside a wavelength of light. Nevertheless, there is reason to be optimistic.

Single photon detection requires a material with a bandgap $E_{g}$ less than $h c / \lambda$. A useful number to keep in mind is that $1 \mathrm{eV}$ corresponds to a wavelength of 1.2398 microns, which is in the infrared.

Fabrication requirements would suggest that it makes sense to go as far into the infrared as far as possible where there are essentially two options, both of which are still in rather early stages of development: 1) superconducting tunnel junctions (STJ's) [17] or 2) pixellated avalanche photodiodes (APD's) [18].

Superconducting tunnel junctions have bandgaps of tens of $\mathrm{meV}$, which correspond to breaking up Cooper pairs. They can, in principle, be sensitive to photons of a small fraction of an electron volt. This would allow pixels of several microns to be used, but to the best of our knowledge this has not yet been done.

Avalanche photodiodes can be used for single photon counting and can also be fabricated as finely pixellated devices. Single photon counting has not yet been demonstrated with position sensitivity, but should certainly be feasible. Bandgaps are reasonable with common semiconductors like silicon and germanium having values less than an electron volt, and thus being sensitive to infrared of wavelengths longer than a micron. Pixellation at the submicron level requires masks made using wavelength of light which are much shorter, but this is in principle possible with ultraviolet light, or with 
shorter wavelengths becoming more and more available at synchrotron light sources.

Quantum efficiencies for both STJ's and APD's can be quite high (in excess of $60 \%$ ), and noise should not be a problem if one uses them in a gated mode with a sufficiently intense correlated photon source. We hope to return to more detailed studies of the feasibility of an experiment along these lines in the near future.

\section{References}

[1] V.P. Nair and A.P. Polychronakos, Phys. Lett. B 505, 267 (2001).

Alain Connes, "Noncommutative Geometry", Academic Press, Harcourt Brace \& Co. Sandiago (1994).

Z. Guralnik, R. Jackiw, S. Y. Pi and A. P. Polychronakos, Phys. Lett. B 517, 450 (2001).

S. Bellucci and A. Nersessian, Phys. Lett. B 542, 295 (2002).

P. Castorina, A.Iorio and D. Zappalà, Noncommutative synchrotron, MIT-CPT 3336.

[2] S. Sivasubramanian, G. Srivastava, A. Vitiello and Y. N. Widom, Phys. Lett. A 311, 97 (2003).

[3] W. Yourgrau and S. Mandelstam, “Variational Principles in Dynamics and Quantum Theory”, Dover Publications Inc., New York, Chapt.13, (1968).

[4] L. Belissard "Ordinary Quantum Hall Effect and Noncommutative Cohomology”, in Proc. on Localization in Disordered Systems, ed. Bad Schandau, Tuebner, Leipzig (1988).

[5] A. Widom and Y. N. Srivastava, arXiv:hep-ph/0111350

[6] Y. N. Srivastava and A. Widom, arXiv:hep-ph/0109020

[7] Y. Srivastava and A. Widom, "Muon $g-2$ Measurements and Non-commutative Geometry of Quantum Beams", Presented at PASCOS2003,Mumbai,India (to appear in PRAMANA).

[8] E.P. Wigner, Ann. Math. 40, 149 (1939).

[9] M.H.L. Pryce, Proc. Ror. Soc. A195, 62 (1948).

[10] T.D. Newton and E.P Wigner, Rev. Mod. Phys. 21, 400 (1949).

[11] A.S. Wightman, Rev. Mod. Phys. 34845 (1962).

[12] J.M. Jauch and C. Piron, Helv. Phys. Acta 40559 (1967).

[13] M. Hawton, Phys. Rev. A 59, 954 (1998).

[14] J. Schwinger “Particles, Sources and Fields”, Percous Books, Reading, Vol. 1, Sec. 1-3, (1998). 
[15] L. Mandel and E. Wolf, "Optical Coherence and Quantum Optics", Cambridge University Press, Cambridge (1995).

[16] G.F. Chew and H.P. Stapp, arXiv:hep-th/0111250

[17] P. Verhoeve, N. Rando, A. Peacock, A. van Dordrecht, A. Poelart, D. J. Goldie and R. Venn, IEEE Trans. on Supercond. 7, 3359 (1997).

[18] Y. Musienko, D. Renker, S. Reucroft, and J. Swain, Study of pixellated high gain avalanche photodiodes for single photon detection, to appear. 